\title{
ON AUSCULTATION OF THE MASTOID PROCESS, WITH REGARD TO SCLEROSIS.
}

\author{
By Dr. Okunnief (St. Petersburg).
}

Read before the Fifth International Congress of Otology, Florence, 1895.

The importance of a solution of the question as to whether, in a case of chronic suppuration, the mastoid process is sclerosed, is obvious to every specialist, and the possibility of detecting it would materially simplify the determination of the treatment. When one has before one's eyes a sufficient means of diagnosing sclerosis, the pathological conditions appear in another light and attain a new value.

High fever, rigors, and sweats must be taken as indications of a serious deep-lying affection, as, for example, thrombosis of the transverse sinus, beginning pyæmia or septicæmia. Further, it must! be remarked that cases of chronic suppurative inflammation of the middle ear run an exceptionally bad course when in their various exacerbations they are complicated by a sclerosis of the mastoid process; they then become dangerous to life. With sclerosis of the mastoid process and obliteration of the cellular structure, the possibility of the pus working its way outwards is done away with, and there disappears every protective barrier which ordinarily holds back the accumulation of pus, and in this way protects the organism for a long time from any serious danger. In sclerosis, the pus in the mastoid cells can neither perforate nor remain in them ; it must find new routes : and as, in such cases, the inflammation is retained in the deeper layers of the temporal bone, the pus must, therefore, make a way for itself towards the sinuses and the meninges.

In view of the fact that in this form of disease pain and infiltration of the soft parts of the mastoid process are absent, the condition dangerous to life may come on almost unnoticed by the surgeon, as well as by the patient. From the absence of pain in the mastoid, as well as in the ear, the patients cannot understand the necessity for an operation, and look upon it as useless. If they agree to it, it is even then with great unwillingness and with the utmost mistrust. In most cases the only indication remaining for the surgeon is the high temperature, which is frequently mistaken for another disease ; as, for example, the influenza of later days. In point of fact, the position of matters becomes distinctly worse. The high temperature is a sign of beginning septicæmia or pyæmia, resulting from the absorption of the products of suppuration in the breaking down clot. It is obvious that in chronic suppurative otitis, with sclerosis of the mastoid, elevation of the temperature is a memento mori, and that the sooner operation is undertaken the more chance is there of a favourable result. On the one hand, we may look upon sclerosis of the mastoid as a natural attempt on the part of the organism to protect itself from the purulent focus lying in its neighbourhood, because, when this goes on normally in the neighbourhood round the 
collection of pus, the danger to life is distinctly diminished. Then purulent suppurations of the middle ear which are complicated with sclerosis of the mastoid, cannot be distinguished by their disturbed course from ordinary cases not so complicated. The difference between the one and the other depends in the first place upon the supervention of elevation of temperature. Those complicated by sclerosis are, in general, characterized by rigors, sweats, headaches, or, at all events, a feeling of weight in the head. The headache is localized most frequently in the neighbourhood of the temples; sometimes the forehead. In the mastoid region there is not the slightest tenderness, nor any infiltration of the soft parts. Some patients will strike their mastoid with the fist in order to prove to their attendant the absence of any local tenderness. Certainly, in some cases there are temporary attacks of pain in the region, but which only last for a short time, and rapidly completely disappear under the action of leeches and blisters.

The only symptom which we have so far is, in most cases, the high pyrexial temperature. Thanks to auscultation of the mastoid process according to my method, we have a valuable indication, and a diagnosis can be quickly made as to the condition of the bones with which we have to deal. In this auscultation one observes that the tone of a tuning fork placed upon the skull is, in case of sclerotic change in the bone, heard louder upon the diseased side than upon the sound one. This is explained by the better conduction of tone through the sclerosed bone as being more compact. Mixed cases of sclerosis-that is to say, those in which, in a mastoid process with sclerosed bony tissue, carious spaces of small size are found-are the most difficult for diagnosis; in such cases repeated examination of the bones is necessary, so that serious attention may be given to the slightest dulness when it is permanent.

A few cases out of a series which I have examined will now be narrated in support of what I have said.

Case I: A soldier, aged twenty-three, suffered with suppuration from the left ear since childhood. The left membrane was opaque, thickened, and had a perforation the size of a pea in the antero-inferior quadrant, the margins of which were hypodermised. The mucous membrane of the cavity was swollen and hypodermic, and there was a considerable discharge of pus through the perforation. The left facial nerve was paralyzed; the right membrana tympana showed nothing abnormal beyond a slight indrawing and opacity.

Treatment began on the 30 th April with irrigations of a 4 per cent. solution of boric acid.

Shortly afterwards, pains began in the left ear, with infiltration and redness of the walls of the meatus, but improvement took place after the application of artificial leeches to the mastoid.

On the 14 th of May there was tenderness of the left mastoid process without infiltration of the soft parts, and the patient complained of rigors. Blisters were applied to the mastoid process, and internally muriate of quinine was administered. Since his entrance into the hospital his temperature was normal, but on the I4th of May it became pyrexial, and the patient complained of rigors, lassitude, diminution of appetite, sleeplessness, and night sweats. The pupils were dilated, but active. After the blisters the tenderness of the mastoid completely disappeared, and forcible percussion occasioned no pain. On the 22nd 


\section{Rhinology, and Otology.}

of May pain was felt at the left angle of the lower jaw in front of the sternomastoid, where, in the depth of the hollow, an affected gland could be felt, of the size of a pigeon's egg. On examination of the bone conduction (auscultation of the mastoid), there was recognized in the upper and lower thirds a clear loud tone from the tuning fork, louder in character than normal, while in the middle third was a slight dulness of the tone.

Diagnosis:-Sclerosis of the mastoid process in its upper and lower thirds. Development of granulation tissue in its depths and thrombosis of the lateral sinus.

Trepanation of the mastoid on May 23rd.

Conditions found on operation: Corresponding to the dulness of tone in the middle third of the mastoid process, at a point two millimètres from the posterior wall of the osseous meatus an opening was made in the bone. This was found to be sclerosed, and yielded to the chisel with great difficulty. The middle and inferior thirds of the process were sclerosed throughout to the extent of more than a centimetre, and the middle third was more than half a centimetre. In the depth of the opening into the bone there was found a mass of granulation tissue bathed in pus, which was cleared away by means of a sharp spoon. After the removal of this, there was found as one reached the sinus a thick layer of thick pus, which welled up with pulsations under the sclerosed upper third of the mastoid. After enlargement of the osseous opening, so that half of the upper third of the mastoid process was removed, one came across the anterior wall of the lateral sinus, which was covered with granulations. Along with the removal of this, the anterior wall of the sinus had to be removed to the extent of a centimetre. The spoon was introduced upwards and downwards along the sinus, so that the thrombus could be no longer seen. Evidently the thrombus, which had already undergone purulent disorganization, had produced a change in the anterior wall of the sinus, so that the pus had made a way for itself to the mastoid process. The wound was cleansed with a mercurial lotion, one half per cent., dusted with iodoform powder, and plugged with iodoform gauze.

(At the present time the patient is still under my treatment.)

Case 2: A soldier, aged twenty-three, had had a purulent discharge from the left ear on and off since childhood. It had broken out again during the last three months. The patient was pale and anæmic. The left tympanic membrane was opaque and thickened, and in the lower segment there was a large perforation. During one and a half months the temperature had been perfectly normal, but then it suddenly rose to $39^{\circ} 5-40 \mathrm{C}$., with rigors and sweats.

The mastoid process showed on its outer surface no departure from normal. There was no tenderness whatever. On auscultation it was found that the sound of the tuning fork along the mastoid process was clearer and louder than on the healthy side. Leeches and fly-blisters produced no beneficial effect.

Diagnosis. - Mastoid sclerosis, thrombosis of the lateral sinus. Trepanation of the mastoid. Conditions found on operation : Throughout there was sclerosis of the mastoid process without a trace of cellular structure. The bone scarcely yielded to the chisel. Nowhere could either pus or granulation tissue be found, and the transverse sinus was not exposed. Communication between the operation wound and the middle ear was established, and an antiseptic bandage applied. On the third day after the operation death took place, and on post-mortem examination there was found thrombosis of the lateral sinus; a purulent breaking down of the thrombus in the vertical portion of the sinus. Septicremia.

Case 3: A Jew, aged twenty-three, who since childhood had had otorrhœa in the left ear. There was a large perforation in the anterior segment of the tympanic membrane, with epidermised margins. After treatment in the hospital for two 
months there suddenly occurred high fever with rigors and night sweats, pain in the whole of the left half of the head, but especially in the left mastoid region. There was no infiltration of the soft parts, and no tenderness behind the ear. On auscultation of the mastoid process the sound was heard more clearly and louder than on the unaffected side. Leeching and blistering were of no use, and trepanation was decided on.

Conditions found on operation : Throughout there was sclerosis of the mastoid process; the bone scarcely yielded to the chisel, and after communication was made with the middle ear an antiseptic dressing was applied. The pains in the head and the mastoid process disappeared immediately after the operation, and the patient got well.

In view of the fact that my method is unknown to many of my confrères here present, I take this opportunity to describe it and to report the results which it has given. I have to ask indulgence for repeating here what I have already published in the Zeitschrift "Bparr, 1893-94," as also in the "Archiv fuir Ohrenheilkunde," Vol. XXXVIII. I investigate the cranial bone conduction of sound, especially through the mastoid process, in the following way: I take an ordinary otoscopic tube with the usual tip at one end, which I introduce into my ear. To the other end I fasten, instead of the ordinary tip, an aural speculum of vulcanite of the smallest possible diameter. I place the latter upon the part of the head concerned, and, in the present instance, upon the different portions of the mastoid process and around it. When I have thus effected junction between the patient and myself, I take a suitable tuning fork ${ }^{-}$, strike it upon a wooden object, and then place its stem upon the middle of the patient's skull, somewhat further forward, below the parietal eminence of the affected side. The investigation carried on in this way gives the following information: When the cranial bones are unchanged the tuning fork gives a clear sound; when, on the other hand, a bone is diseased, the tone is duller over the seat of the pus or softened bone; on the unaffected mastoid process the tone is as clear as on the neighbouring parts of the skull. On auscultation of a sclerosed mastoid the tone conducted through the skull is always clearer and more audible than on the unaffected sides; hence the sclerosed bone is distinguished from the normal by its increased power of conducting tone.

Dundas Grant (Trans.).

\title{
CAYERNOUS ANGIOMA OF THE EAR.
}

\author{
By Dr. E. J. Moune (Bordeaux). \\ Read before the International Congress of Otology, Florence, 1895.
}

"IF one examines histologically a large number of aural polypi," writes Prof. Politzer, "we meet with two principal forms-round cell-polypi " and fibromata." A little further on he adds : "Some polypi of the tym" panic cavity are traversed by numerous blood vessels, and these acquire " the character of cavernous polypi, or angiomata. According to the

1 I have always used a tuning fork of 256 vibrations, set vibrating by the introduction of a rod of steel between the two blades which converge towards their points, the steel rod being pulled out from between them. Deeper tuning forks than this give so powerful a tone that they are heard througt the otherear by air conduction, and confusion results from the sound being beard in this double manner. Higher tuning forks are also unsatisfactory, as their short lasting tone comes too quickly to an end. It is important for every otologist to practise himself in this auscultation of the mastoid process. 